\title{
MEASURES ASSOCIATED TO CHERN, LEVINE AND NIRENBERG NORMS
}

\author{
MORRIS KALKA ${ }^{1}$
}

\begin{abstract}
We define an intrinsic outer measure on complex submanifolds of a given complex manifold. This is modeled on the norm on the real homology defined by Chern, Levine and Nirenberg.
\end{abstract}

Here we extend the definition of the intrinsic norm on the real homology groups of a complex manifold, defined by Chern, Levine and Nirenberg [1]. We begin by recalling the definition of these norms.

Let $M$ be a complex manifold and $d^{c}=i(\bar{\partial}-\partial)$ the imaginary part of the exterior differentiation operator $d$. Let $\mathscr{F}=\left\{u \in C^{2}(M) \mid u\right.$ plurisubharmonic, $0<$ $u<1\}$. If $\gamma \in H_{p}(M, \mathbf{R})$ denotes a $p$-dimensional homology class we define

$$
N(\gamma)= \begin{cases}\sup _{u \in \mathscr{F}} \inf _{T \in \gamma} T\left|d^{c} u \wedge\left(d d^{c} u\right)^{k-1}\right|, & p=2 k-1, \\ \sup _{u \in \mathscr{F}} \inf _{T \in \gamma} T\left|d u \wedge d^{c} u \wedge\left(d d^{c} v\right)^{k-1}\right|, & p=2 k,\end{cases}
$$

where $T$ runs over the class of all currents (in the sense of deRham) of $\gamma$.

$N$ is a norm in the sense that $N(\gamma) \geqslant 0, N(a \gamma)=|a| N(\gamma)$ and $N$ satisfies the triangle inequality $N\left(\gamma_{1}+\gamma_{2}\right) \leqslant N\left(\gamma_{1}\right)+N\left(\gamma_{2}\right)$. From the point of view of holomorphic mappings, the most important property of $N$ is that it does not increase under holomorphic mappings. In fact, if $f: M_{1} \rightarrow M_{2}$ is holomorphic and $f_{*}: H_{p}\left(M_{1}, \mathbf{R}\right) \rightarrow H_{p}\left(M_{2}, \mathbf{R}\right)$ is the map induced on homology, then $N_{M_{2}}\left(f_{*} \gamma\right) \leqslant$ $N_{M_{1}}(\gamma)$.

Suppose now that $S \subset M$ is a complex submanifold. We can extend the definition of $N$ from homology classes to an outer measure on complex submanifolds in the following way.

DeFINITION 1. $\mu_{N}(S)=\sup _{u \in \mathcal{F}} \int_{S} d u \wedge d^{c} u \wedge\left(d d^{c} u\right)^{p-1}$.

Our main result will be that a relatively compact subset of a complex submanifold has finite outer measure in the sense of our definition.

There is no natural reason to restrict our definition to complex submanifolds. However, in this case, it is reasonable to restrict the class $\mathscr{F}$, as we now explain. If $\gamma$ is a homology class, it is clearly sufficient to consider functions $u$ for which the

Received by the editors August 24, 1982.

1980 Mathematics Subject Classification. Primary 32H15.

${ }^{1}$ Research supported by an NSF grant. 
corresponding differential forms are closed. Hence we are led to the classes

$$
\begin{array}{ll}
\tilde{\mathscr{F}}_{p}=\mathscr{F} \cap\left\{u \mid\left(d d^{c} u\right)^{k}=0\right\}, & p=\operatorname{dim} \gamma=2 k-1, \\
\tilde{\mathscr{F}}_{p}=\mathscr{F} \cap\left\{u \mid d u \wedge\left(d d^{c} u\right)^{k}=0\right\}, & p=\operatorname{dim} \gamma=2 k .
\end{array}
$$

Thus for $S$ a submanifold which is not necessarily complex, we define the function $\tilde{\mu}_{M}(S)$ analogously to the definition above.

DEFinition 2. If $S \subset M$ is a submanifold of the complex manifold $M$, then we define

$$
\tilde{\mu}_{M}(S)= \begin{cases}\sup _{u \in \tilde{\mathscr{F}}_{p}} \int_{S} d^{c} u \wedge\left(d d^{c} u\right)^{p-1}, & \operatorname{dim} S=2 p-1, \\ \sup _{u \in \tilde{\mathscr{F}}_{p}} \int_{S} d u \wedge d^{c} u \wedge\left(d d^{c} u\right)^{p-1}, & \operatorname{dim} S=2 p .\end{cases}
$$

We note that if $S$ is a complex submanifold, then $\tilde{\mu}_{M}(S) \leqslant \mu_{M}(S)$. Our main result is

THEOREM 1. If $\tilde{S} \subset M$ is a complex p-dimensional submanifold of the complex manifold $M$ and $S$ is a relatively compact open submanifold of $\tilde{S}$, then $\mu_{M}(S)<\infty$.

Proof. If $u \in \mathcal{F}$, then $u \mid \tilde{S}$ is plurisubharmonic on $\tilde{S}$. Let $\rho \in C_{0}^{\infty}(\tilde{S})$ have compact support in $\tilde{S}$ and satisfy

(i) $0 \leqslant \rho \leqslant 1$,

(ii) $\rho \equiv 1$ on $S$.

Then

$$
\left|\int_{S} d u \wedge d^{c} u \wedge\left(d d^{c} u\right)^{p-1}\right| \leqslant\left|\int_{\tilde{S}} \rho d u \wedge d^{c} u \wedge\left(d d^{c} u\right)^{p-1}\right| .
$$

If we integrate by parts we see that the integral on the right is equal to

$$
\begin{aligned}
\mid-\int_{\tilde{S}} u d \rho \wedge & d^{c} u \wedge\left(d d^{c} u\right)^{p-1}-\int_{\tilde{S}} u \rho\left(d d^{c} u\right)^{2 p} \mid \\
\leqslant & \left|-\frac{1}{2} \int_{\tilde{S}} d \rho \wedge d^{c} u^{2} \wedge\left(d d^{c} u\right)^{p-1}\right|+\left|\int_{(\operatorname{supp} \rho)}\left(d d^{c} u\right)^{p}\right| \\
& \leqslant \frac{1}{2}\left|\int_{(\operatorname{supp} \rho)} d d^{c} \rho \wedge\left(d d^{c} u\right)^{p-1}\right|+\left|\int_{(\operatorname{supp} \rho)}\left(d d^{c} u\right)^{p}\right|
\end{aligned}
$$

Each of these integrals is finite independent of $u$ by the following result of [1].

LEMMA. Let $u$ be a $C^{2}$ plurisubharmonic function on the polydisc $\left\{z \in \mathbf{C}^{n}|| z^{i} \mid<r_{i}\right\}$ with $0<u<1$. Then $\exists B$ independent of $u$ such that for any $r \times r$ minor $V$ of $\left(\partial^{2} u / \partial z^{i} \partial \bar{z}^{j}\right)$

$$
\int_{\left|z^{i}\right| \leqslant p_{i}<r_{i}}\left(|\operatorname{det} V|+\sum_{k}\left|\frac{\partial u}{\partial z^{k}}\right|^{2}\right) \leqslant B
$$


We do not know whether our result is true when $S$ is not complex. We suspect not. There are cases, however, when $\tilde{\mu}(M)<\infty$.

CorollaRY. Suppose $H_{p}(M, \mathbf{R})=0$ and $S \subset M$ is a p-dimensional submanifold of $M$ with the property that $\partial S=\partial K$ where $K$ is open and relatively compact in a complex submanifold $V$ of $M$. Then $\tilde{\mu}_{M}(S)<\infty$.

Proof. By the differential equation and Stokes' Theorem, for any $u \in \tilde{\mathscr{F}}_{p}$,

$$
\int_{S} d u \wedge d^{c} u \wedge\left(d d^{c} u\right)^{p-1}=\int_{K} d u \wedge d^{c} u \wedge\left(d d^{c} u\right)^{p-1}
$$

for all $u \in \tilde{\mathscr{F}}_{p}$, since $\int_{S \cup K} d u \wedge d^{c} u \wedge\left(d d^{c} u\right)^{p-1}=0$.

REMARKS. (1) $\tilde{\mu}_{M}$ cannot be made into a monotone outer measure on general submanifolds. By monotone we understand $A \subset B \Rightarrow \mu(A) \leqslant \mu(B)$. This is impossible for if $M$ is contractible and $K \subset V$ is an open relatively compact subset in the complex submanifold $V$, with $\tilde{\mu}_{M}(V)>0$, we can form $\tilde{K}$, a compact smooth manifold with $K \subset \tilde{K}$. Of course $\tilde{\mu}_{M}(\tilde{K})=0$.

(2) One can define $\mu_{M}$ on a subvariety by integrating over the regular points. In general we do not know if $\mu_{M}$ is finite. However, if we can resolve the singularity (e.g., if the singularity is locally algebraic), then we can conclude that $\mu_{M}$ is finite. The reason for this is that, by definition, if $f: M_{1} \rightarrow M_{2}$ is holomorphic, then $\mu_{M_{2}}(f(V)) \leqslant \mu_{M_{1}}(V)$ for any submanifold $V$. Thus if $(\tilde{M}, \tilde{V}, \pi)$ is a resolution of the singularity of the subvariety $V \subset M$ and if $K \subset V$ is compact since $\pi$ is proper, $\mu_{M}(K) \leqslant \mu_{\tilde{M}}\left(\pi^{-1}(K)\right)<\infty$.

(3) Of course the major problem left here is to compute examples. One would like to know for complex submanifolds $V$ of the unit ball in $\mathbf{C}^{n}$, how rapidly does $\mu\left(V \cap\left\{|z|^{2}<r\right\}\right) \rightarrow \infty$ as $r \rightarrow 1$ ?

\section{BIBLIOGRAPHY}

1. S. S. Chern, Harold I. Levine and L. Nirenberg, Intrinsic norms on a complex manifold. Global Analysis, Univ. of Tokyo Press, Tokyo, 1969, pp. 119-139.

Department of Mathematics, Tulane University, New Orleans, Louisiana 70118 